\title{
Perturbative Approach to the Bak-Sneppen Model
}

\author{
M. Felici, ${ }^{1,2}$ G. Caldarelli, ${ }^{1}$ A. Gabrielli, ${ }^{1,2}$ and L. Pietronero ${ }^{1}$ \\ ${ }^{1}$ INFM-Unità di Romal and Dipartimento Fisica, Università "La Sapienza," Piazzale Aldo Moro 2, 00185, Roma, Italy \\ ${ }^{2}$ Laboratoire de la Physique de la Matière Condensée, Ecole Polytechnique, 91128 Palaiseau, France
}

(Received 27 October 2000)

\begin{abstract}
We study the Bak-Sneppen model in the probabilistic framework of the run time statistics (RTS). This model has attracted a large interest for its simplicity being a prototype for the whole class of models showing self-organized criticality. The dynamics is characterized by a self-organization of almost all the species fitnesses above a nontrivial threshold value, and by a lack of spatial and temporal characteristic scales. This results in avalanches of activity power law distributed. In this Letter we use the RTS approach to compute the value of $x_{c}$, the value of the avalanche exponent $\tau$, and the asymptotic distribution of minimal fitnesses.
\end{abstract}

DOI: $10.1103 /$ PhysRevLett.86.1896

The concept of self-organized criticality has been introduced in order to explain the ubiquitous presence of scale free phenomena in nature. Ranging from fractures [1] to river basins [2], the lack of spatial and/or temporal characteristic scales is the main feature of these processes [3]. We focus here on the Bak-Sneppen (BS) model [4] of interacting species in an ecosystem. We develop a perturbative approach to BS based on the run time statistics (RTS) $[5,6]$. We evaluate the main quantities for the model in the stationary state: the fitnesses histogram, the distribution of minimal fitnesses, and the avalanche exponent.

The BS model is defined as follows: a 1D ring of $N$ different sites models the $N$ species present in the ecosystem. Links between first nearest neighbor (fnn) sites model interaction between species (i.e., predation). A random number $x_{i} \in[0,1]$, extracted from the uniform probability density function (pdf) $f_{0}(x)=1$, is assigned to each site/species $i$ in the system. The $x_{i}$ 's quantify the fitness of species $i$ to survive the competition with the other species in the environment. At each time step, the species with the minimal fitness is selected and removed. The species connected to it through predation (i.e., the fnn) are also removed, regardless of their fitness. Three new species with randomly extracted fitnesses take their place (refreshing rule). After a transient period this system selforganizes in a stationary state characterized by two main features: (1) the normalized fitnesses histogram $\phi(x)$ is given, apart from corrections of order $1 / N$, by $\phi(x)=$ $\theta\left(x-x_{c}\right) /\left(1-x_{c}\right)$, where $\theta(x)$ is the usual step function, and $x_{c}=0.66702(3)$ [7]; (2) the stationary dynamics evolves as a sequence of critical avalanches [4,8,9], the duration $s$ of which is power law distributed: $P(s) \sim s^{-\tau}$ with $\tau \simeq 1.07$. The best analytical results for BS come from mean-field approaches giving a threshold $x_{c}=1 / 3$ [10], very far from the real numerical value. This striking disagreement led us to investigate if the actual value can be computed by using a theoretical tool, called RTS [5,6]. RTS is designed to study growth processes in a medium with quenched disorder. In particular, the main results $[11,12]$ obtained through RTS deal with the class of mod-
PACS numbers: 05.65.+b, 02.50.-r, 64.60.Ak, 87.23.Kg

els derived by the invasion percolation (IP) in $d=2$ [13], to which the BS model can be related. RTS is based on the intuitive idea that the larger the number of time steps one species survives, then probably the larger its fitness is. In other words, if at the first time step with no information, one assigns a uniform pdf for the species fitnesses, at successive time steps information about the history increases the effective pdf at high fitness values for the surviving species. More formally, this information is stored in effective (conditional) pdf's $\left\{f_{i, t}(x)\right\}$ of the quenched disorder variables $\left\{x_{i}\right\}$. These effective pdf's can then be used to develop a step by step algorithm to describe probabilistically the whole tree of the possible dynamics of the system, starting from the last available situation [6]. Let us suppose to know at time $t$ the set of effective pdf's $\left\{f_{i, t}(x)\right\}$. By using joint probability, it is possible to write for each species the effective probability to be selected at that time step, and one can update the effective pdf's, obtaining the new set of functions $\left\{f_{i, t+1}(x)\right\}$. For example, consider a limit case with a system composed only by two sites $a, b$. The minimum is selected and refreshed without affecting the other. At the beginning $(t=0)$ both pdf's are uniform and equal to 1 , and the probabilities $\mu_{a, 0}, \mu_{b, 0}$ to be selected for sites $a, b$ are both equal to $1 / 2$. We assume without loss of generality that $x_{a}<x_{b}$. By using the postulate of the theory of conditioned probability

$$
\begin{aligned}
& f_{a, 1}\left(x_{a}\right)=1, \\
& f_{b, 1}\left(x_{b}\right)=\frac{f_{b, 0}\left(x_{b}\right)}{\mu_{a, 0}} \int_{0}^{x_{b}} d x_{a} f_{a, 0}\left(x_{a}\right)=2 x_{b},
\end{aligned}
$$

and consequently

$$
\begin{aligned}
& \mu_{a, 1}=\int_{0}^{1} d x_{b} f_{b, 1}\left(x_{b}\right) \int_{0}^{x_{a}} d x_{a} f_{a, 1}\left(x_{a}\right)=2 / 3, \\
& \mu_{b, 1}=\int_{0}^{1} d x_{a} f_{a, 1}\left(x_{a}\right) \int_{0}^{x_{a}} d x_{b} f_{b, 1}\left(x_{b}\right)=1 / 3 .
\end{aligned}
$$

That is, the just refreshed site has a larger probability to grow. This argument can be easily generalized for any 
time $t$. For instance, if the site selected is always the same, $f_{b, t}=(t+1)\left(x_{b}\right)^{t}$ and $\mu_{b, t}=\frac{1}{t+2} \simeq \frac{1}{t}$.

In order to implement this program for the BS, one needs only an initial site configuration with known $\left\{f_{i, t}(x)\right\}$. There are two possibilities: (i) to follow the dynamics from the first time step $t=0$, having obviously $f_{i, 0}(x) \equiv$ $f_{0}(x)=1$ for each $i$; (ii) to start at an arbitrary time $t$ for which the set $\left\{f_{i, t}(x)\right\}$ is known. In the following, we consider mainly the case (ii): the system is considered already at the critical stationary state, just after the first step of a critical avalanche. We call the site selected at this first step the initiator of the avalanche. We recall that the initiator fitness [9] lies in an interval of order $1 / N$ around $x_{c}$, and all the other sites have a fitness $x>x_{c}$. Avalanches are defined as geometrically and causally connected sequences of growths. Since it has been proved that each avalanche is independent of the others, the probabilistic study of a generic critical avalanche provides a complete statistical description of the stationary state. Without loss of generality, we consider the initiator placed at $i=0$ and its selection at $t=0$. At $t=1$, the just grown initiator and its two fnn sites have the fitness uniformly distributed because of the refreshing rule [i.e., $f_{0,1}(x)=f_{-1,1}(x)=$ $\left.f_{1,1}(x)=f_{0}(x)=1\right]$. Let us call $A_{t}$ (active sites) the set of sites refreshed by the avalanche up to its $t$ th step. Consequently, $A_{1}$ is composed of the three sites $-1,0$, and +1 . The set $A_{t}$ defines a connected segment on the system because of the geometrical connectivity of an avalanche. One can represent the evolution of the system as a branching process (see Fig. 1). At each time step, the growth of each active site corresponds to a branching event.

The growths of "nonactive" sites are not included in the branching tree, because this implies the end of the avalanche and the beginning of a new one. Any finite connected path on this branching tree represents a single realization of the avalanche. The tree contains all the possible realizations of the avalanche with a given initiator.

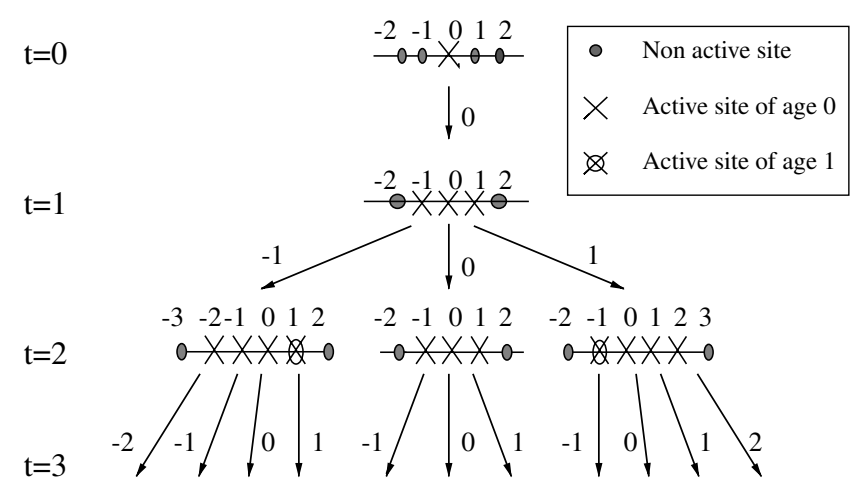

FIG. 1. The first three temporal steps of an avalanche tree. The initiator $i=0$ is selected at $t=0$ and the possible growths are shown.

The avalanche proceeds, after the selection of the initiator, if at least one of the three sites belonging to $A_{1}$ has fitness below $x_{c}$. In general, at the $t$ th time step of the avalanche, if one knows the effective pdf $f_{i, t}(x)$ for each site $i \in A_{t}$, one can write the effective growth probability of site $i$ conditioned to the fixed past history:

$$
\mu_{i, t}=\int_{0}^{x_{c}} d x_{i} f_{i, t}\left(x_{i}\right) \prod_{j \neq i}\left[\int_{x_{i}}^{1} d y f_{j, t}(y)\right] .
$$

Each of these possible growth events corresponds to a branch of the tree leading from the starting configuration to a new one characterized by a new set $A_{t+1}$ of active sites with their new effective pdf's. The set of sites $A_{t+1}$ is related with the "mother" set $A_{t}$. If the just grown site $i$ is not an extreme of the segment defined by $A_{t}$, then $A_{t+1} \equiv A_{t}$. If, instead, $i$ is the left (right) extreme of $A_{t}$, then the left (right) fnn of $i$ must be added to $A_{t}$ in order to obtain $A_{t+1}$. After the selection of site $i$, the new effective pdf's of the sites belonging to $A_{t+1}$ are obtained from those of $A_{t}$ by applying both the refreshing rule of the dynamics and the law of conditional probability $P(B \mid A)=\frac{P(A \cap B)}{P(A)}$ to store the information about the last growth event:

$$
\begin{aligned}
& f_{k, t+1}(x)= \begin{cases}\frac{f_{k, t}(x)}{\mu_{i, t}} \int_{0}^{x} d x_{i} f_{i, t}\left(x_{i}\right) \prod_{j}\left[\int_{x_{i}}^{1} d y f_{j, t}(y)\right] & x \leq x_{c}, \\
f_{k, t}\left(x_{c}\right) & x>x_{c},\end{cases} \\
& f_{i, t+1}(x)=f_{i-1, t+1}(x)=f_{i+1, t+1}(x)=f_{0}(x)=1,
\end{aligned}
$$

where $k$ is any species in the set $A_{t}$ different from $i$ and its neighbors, and $j$ is an active site different from $k, i$. The pdf of an active site is always constant above $x_{c}$ because in the stationary state no site with $x>x_{c}$ grows, then no information is available for $x>x_{c}$. Through this algorithm one can store step by step information on the fitnesses from the actual fixed history of the avalanche. By iterating Eqs. (3) and (4), one can write for a time length $\Delta t$ the probability of any connected path $C(\Delta t)$ belonging to the tree as

$$
W[C(\Delta t)]=\prod_{t=0}^{\Delta t} \mu_{i_{t}, t},
$$

where $i_{t}$ is a site growing at time $t$ th of the path $C(\Delta t)$.

Let us now write an equation for the average fitness histogram $\phi_{t}(x)$. From the refreshing rule, one has

$$
\begin{aligned}
N \phi_{t+1}(x)= & N \phi_{t}(x)-m_{i, t+1}(x)-\hat{f}_{i-1, t+1}(x) \\
& -\hat{f}_{i+1, t+1}(x)+3
\end{aligned}
$$

where $m_{i, t+1}(x), \hat{f}_{i-1, t+1}(x)$, and $\hat{f}_{i+1, t}(x)$ are the effective pdf's that sites $i, i-1$, and $i+1$ would have, respectively, after the selection of site $i$, if their fitnesses were not refreshed. Equation (6) states that three species are removed with their pdf's, and three new species with uniform pdf's enter. Considering the average $\langle\cdots\rangle$ of Eq. (6) over all 
the possible paths, and imposing the stationarity condition $\phi_{t+1}(x)=\phi_{t}(x)=\phi(x)$, one has

$$
\left\langle m_{i}(x)\right\rangle+\left\langle\hat{f}_{i+1}(x)+\hat{f}_{i-1}(x)\right\rangle=3 .
$$

We use Eq. (7) to estimate $x_{c}$ and the behavior of $\phi(x)$ under the simple hypothesis that all the sites not touched by the avalanche have a fitness $x>x_{c}$ (which comes from the stationarity condition). The functions $\hat{f}_{i-1, t+1}(x)$ and $\hat{f}_{i+1, t+1}(x)$ are given by the second line of Eq. (4) with $k=i-1$ and $k=i+1$, respectively. In order to perform the average $\langle\cdots\rangle$, one has to consider all the ways in which the selection of site $i$ represents the $t$ th step of an avalanche (i.e., one has to consider all the finite paths of the tree of Fig. 1). Then one can write

$$
\begin{aligned}
& \left\langle\hat{f}_{i-1}(x)+\hat{f}_{i+1}(x)\right\rangle \\
& =\frac{\sum_{t} \sum_{C(t)} W[C(t)]\left[\hat{f}_{i-1, t+1}(x)+\hat{f}_{i+1, t+1}(x)\right]_{C(t)}}{\sum_{t} \sum_{C(t)} W[C(t)]},
\end{aligned}
$$

where $W[C(t)]$ is the RTS statistical weight of the generic path $C(t)$ of length $t$, in the tree of Fig. 1, given by Eq. (5). Moreover $[\cdots]_{C(t)}$ indicates that the functions inside brackets are evaluated by applying step by step the RTS algorithm to the path $C(t)$. Note, anyway, that if $i$ is the left (right) extreme of $A_{t}$, then the knowledge of the path does not give any information about the sites $i-1$ $(i+1)$ apart from, before refreshment, $x_{i-1}>x_{c}\left(x_{i+1}>\right.$ $\left.x_{c}\right)$. In this case we can approximate $\left[\hat{f}_{i-1, t+1}(x)\right]_{C(t)}=$ $\phi(x)\left\{\left[\hat{f}_{i+1, t+1}(x)\right]_{C(t)}=\phi(x)\right\}$. Distinguishing these last terms in $\phi(x)$ in Eq. (8) from the others, one can write

$$
\left\langle\hat{f}_{i-1}(x)+\hat{f}_{i+1}(x)\right\rangle=A\left(x_{c}\right) \phi(x)+B\left(x, x_{c}\right),
$$

where $A\left(x_{c}\right)$ and $B\left(x, x_{c}\right)$ are positive functions, and $B\left(x, x_{c}\right)=B\left(x_{c}, x_{c}\right)$ for $x>x_{c}$, because of Eq. (4).

In principle, we should evaluate $\left\langle m_{i}(x)\right\rangle$ applying the rule of conditional probability in analogy with Eq. (4), and then performing the average as in Eq. (8). However, this would reduce Eq. (7) to a trivial identity. For this reason, in order to evaluate $\left\langle m_{i}(x)\right\rangle$, we adopt another strategy in the optics of the point (i). We impose that $x_{i}$ is the minimal fitness in the whole system at a certain time step $T \gg N$ from the actual beginning of the dynamics. Consequently, we apply the RTS algorithm from the first time step [6] of the dynamics up to $T$ (in this case we have to consider all the system sites). In the end, we consider the average $\langle\cdots\rangle$ over all the possible histories up to time $T$. Since for $T \gg N$ the system must be in the stationary state [9], then, after the average, this description must be consistent with the previous one where the system was considered directly in the stationary state. In order to simplify the mathematical treatment, we use the formula obtained for the generalized $\beta$-BS model [14], and we take the limit $\beta \rightarrow \infty$ at the end of calculations:

$$
\left\langle m_{i}(x)\right\rangle=N \phi(x) \theta\left(x_{c}-x\right),
$$

where $x_{c}$ is defined by the relation $e^{-\beta x_{c}}=$ $\int_{0}^{1} d x \phi(x) e^{-\beta x}$ with $\beta \gg 1$, which gives also a deeper meaning to $x_{c}$ [14]. Note that in this contest the presence of a finite threshold $x_{c}$ arises naturally, without external assumption. In order to obtain Eq. (10), we used the central limit theorem for $N \gg 1$ [14] and we made the following approximation [15]:

$$
\left\langle\prod_{j=1}^{N} f_{j, T}\left(x_{j}\right)\right\rangle \simeq \prod_{j=1}^{N}\left\langle f_{j, T}\left(x_{j}\right)\right\rangle=\prod_{j=1}^{N} \phi\left(x_{j}\right) .
$$

Introducing Eqs. (9) and (10) in Eq. (7), we obtain

$$
\phi(x)=\frac{3-B\left(x, x_{c}\right)}{A\left(x_{c}\right)+N \theta\left(x_{c}-x\right)} .
$$

Note that, because of the behavior of $B\left(x, x_{c}\right), \phi(x)$ is constant for $x>x_{c}$, while for $x<x_{c}$ is of the order of $1 / N$. There is only one value of $x_{c}$ for which $\phi(x)$ is normalized [i.e., $\int_{0}^{1} \phi(x) d x=1$ ]. This value gives the value $x_{c}$ of the BS threshold.

In principle, one should consider the contribution to Eq. (8) coming from any length $t$ in the avalanche tree (Fig. 1). By stopping the sums at a value $t=n$, we obtain an $n$-order approximation. We have evaluated the probability of the paths, through RTS, up to $n=7$ by using a computer program for numerical integration.

The results for $x_{c}$, for $n=2$ up to $n=7$, are reported in Fig. 2: The best evaluation is $x_{c}(n=7) \simeq 0.465$, much better than the mean-field result $x_{c}=1 / 3$, but still quite far from the above reported numerical value. However, we verified that this behavior is compatible with an asymptotic value $x_{c}(n \rightarrow \infty) \simeq 0.66$. To this aim, we made a fit with the simplest possible function $x_{c}(n)=0.66-a x^{b}$ (Fig. 2) which is well compatible with the given asymptotic value. The fit values are $a=0.291 \pm 0.003$ and

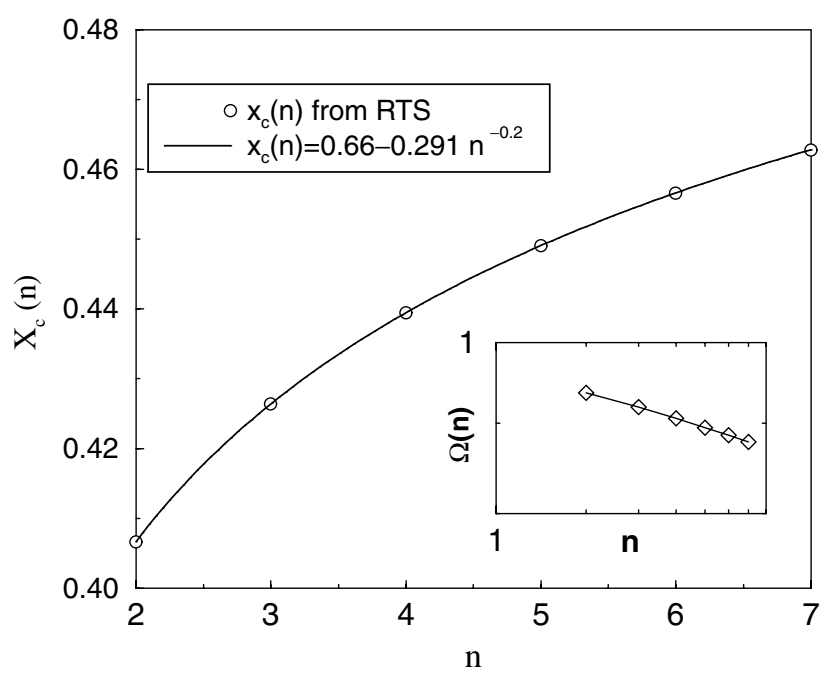

FIG. 2. Empty points represent the values of $x_{c}$ obtained by the application of the RTS algorithm from $n=2$ to $n=7$. The continuous line represents the fit curve $x_{c}(n)=0.66-$ $a x^{b}$ with $a=0.291 \pm 0.003$ and $b=0.20 \pm 0.03$. The inset shows the behavior of $\Omega(n)$ up to $n=7$. Assuming $\Omega(n) \sim$ $n^{-\tau+1}$ also for small $n$, one finds $\tau \simeq 1.05$. 


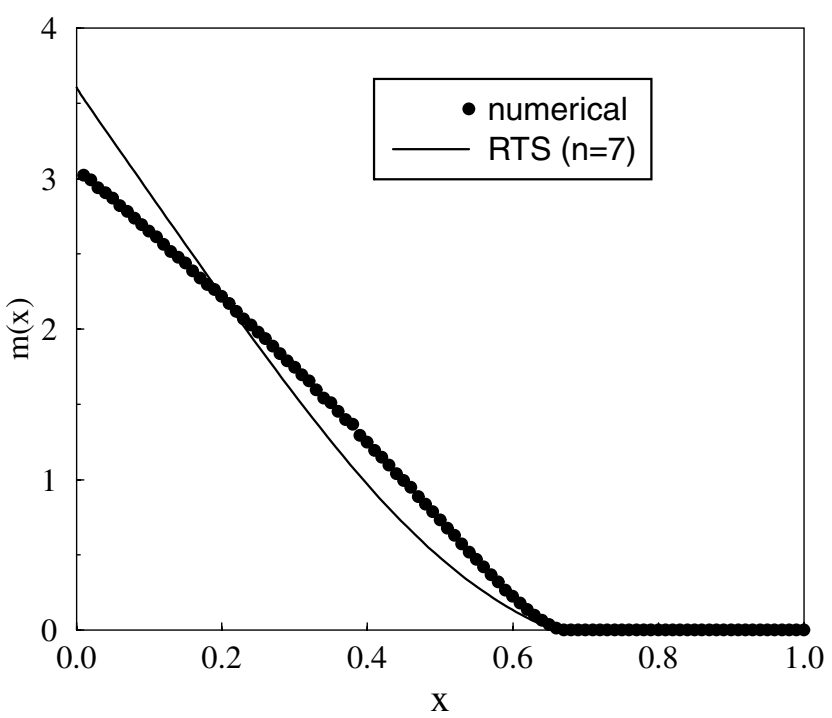

FIG. 3. The continuous line gives the theoretical stationary distribution $m(x)$ of the minimal fitnesses where $n=7$ and assuming $x_{c}(n \rightarrow \infty)=0.66$. The points represent the numerical behavior evaluated in extensive simulations.

$b=0.20 \pm 0.03$. This small value of $b$ is due to the fact that the avalanche size distribution $P(s)$ [9] is characterized by a small exponent $(\tau \simeq 1.07)$, henceforth all the sizes $s$ are important for statistics. One can use $x_{c}(n \rightarrow \infty)$ to evaluate both the avalanche exponent $\tau$ and the average minimum distribution $m(x) \equiv\left\langle m_{i}(x)\right\rangle$. The exponent $\tau$ can be found studying, using $x_{c}(n \rightarrow \infty)=0.66$ in the Eqs. (3) and (4), the behavior of $\Omega(t)=\sum_{C(t)} W[C(t)]$ for $t$ ranging from 1 to the maximal $n$ possible. Indeed $\Omega(t)$ is proportional to the probability that the avalanche lasts at least $t$ time steps. In the scaling regime, $\Omega(t) \sim$ $t^{-\tau+1}$. Making this hypothesis, the result for $n=7$ is $\tau(n=7) \simeq 1.05$ (see inset in Fig. 2), which is in agreement with the known numerical value. Clearly, the hypothesis of the scaling regime for $n=7$ is a strong hypothesis, but it is based on the fact that for IP, which has similar dynamical rules, it has been shown [11] that the microscopical dynamical rules are already the scale invariant ones. Finally, we can obtain an approximation of the distribution of the minimal fitness $m(x)$ in the stationary state considering Eqs. (10) and (11), where the latter one is evaluated using RTS up to $n=7$, but using $x_{c}(n \rightarrow \infty)$ in the RTS calculations.

Imposing normalization, we obtain the function $m(x)$ reported in Fig. 3. In the same figure this result is compared with the known asymptotic numerical distribution of minimal fitnesses [4]. The agreement is again quite good, considering the strong approximation in truncating the sums of Eq. (8) at $n=7$. Finally, it is worth noting that our method includes also the mean-field results for BS. We recall that [10] in the mean-field version of the model, when a site $i$ is selected, the other two sites refreshed are not the fnn sites, but two randomly chosen system sites. This means that in Eq. (7) one has to use $\left\langle f_{i-1}(x)\right\rangle=\left\langle f_{i+1}(x)\right\rangle=\phi(x)$. Henceforth, the asymptotic histogram becomes

$$
\phi(x)=\frac{3}{2+N \theta\left(x_{c}-x\right)},
$$

that is $\phi(x)=O(1 / N)$ for $x<x_{c}$ and $\phi(x)=3 / 2$ for $x>x_{c}$. Normalizing $\phi(x)$, one gets $x_{c}=1 / 3$, which is the above cited mean-field result.

In conclusion, this paper presents a kind of perturbative approach to the BS model, based on the probabilistic framework called run time statistics. Through this approach, we compute the self-organized threshold $x_{c}$, the avalanche exponent $\tau$, and the stationary distribution of minimal fitnesses $m(x)$. In principle one could also obtain in this way the exponent $\mu$ characterizing the sites covered by an avalanche. However, since we can reach at best the 7 th time step of growth, this results in a very poor statistics for the number of points covered by the paths.

The authors acknowledge the support of the EU Network ERBFMRXCT980183.

[1] A. Petri, G. Paparo, A. Vespignani, A. Alippi, and M. Costantini, Phys. Rev. Lett. 73, 3423 (1994).

[2] G. Caldarelli, A. Giacometti, A. Maritan, I. RodriguezIturbe, and A. Rinaldo, Phys. Rev. E. 55, R4865 (1997).

[3] P. Bak, C. Tang, and K. Wiesenfeld, Phys. Rev. Lett. 59, 381 (1987); Phys. Rev. A 38, 364 (1988).

[4] P. Bak and K. Sneppen, Phys. Rev. Lett. 71, 4083 (1993); H. Flyvbjerg, K. Sneppen, and P. Bak, Phys. Rev. Lett. 71, 4087 (1993).

[5] L. Pietronero and W. R. Schneider, Physica (Amsterdam) 119A, 249 (1990).

[6] M. Marsili, J. Stat. Phys. 77, 733 (1994); A. Gabrielli, M. Marsili, R. Cafiero, and L. Pietronero, J. Stat. Phys. 84, 889 (1996).

[7] P. Grassberger, Phys. Lett. A 200, 277 (1995).

[8] M. Marsili, Europhys. Lett. 28, 385 (1994).

[9] M. Paczuski, S. Maslov, and P. Bak, Phys. Rev. E 53, 414 (1996).

[10] J. de Boer, B. Derrida, H. Flyvbjerg, A. D. Jackson, and T. Wettig, Phys. Rev. Lett. 73, 906 (1994).

[11] A. Gabrielli, R. Cafiero, M. Marsili, and L. Pietronero, Europhys. Lett. 38, 491 (1997); R. Cafiero, A. Gabrielli, and M. Marsili, Phys. Rev. E 55, 7745 (1997).

[12] R. Cafiero, G. Caldarelli, and A. Gabrielli, Phys. Rev. E 56, R1291 (1997); R. Cafiero, A. Gabrielli, M. Marsili, L. Pietronero, and L. Torosantucci, Phys. Rev. Lett. 79, 1503 (1997); G. Caldarelli, R. Cafiero, and A. Gabrielli, Phys. Rev. E 57, 3878 (1998).

[13] D. Wilkinson and J.F. Willemsen, J. Phys. A 16, 3365 (1983).

[14] A. Gabrielli, G. Caldarelli, and L. Pietronero, Phys. Rev. E 62, 7638 (2000).

[15] Note that, in this case, the functions $f_{j, t}(x)$ should be obtained by applying the above mentioned strategy (i) following the dynamics from the real beginning and not directly in the stationary state. 